\title{
Child abuse: the role of psychiatrists
}

\author{
Asiri Rodrigo, Nisansala Liyanage
}

Child maltreatment is increasingly being recognised as an important public health issue in Sri Lanka. Psychiatrists in Sri Lanka have a central role to play in aspects of prevention, recognition, assessment and management of child maltreatment.

Child abuse can be defined as acts of omission or commission in interactions between adults or adolescents and children that have caused, or are likely to cause, injury, death or emotional harm to the child (1). They include neglect, physical abuse, emotional abuse, sexual abuse and fabricated or induced illness. It is believed that more than 40 million children worldwide are abused each year (1). Prevalence of child abuse has been recorded to be as high as $53 \%$ while sexual abuse rates ranged from 23.5 to $27.5 \%$ in females (2). Studies show child abuse occurs within all cultures, ethnicities and religions, at every socioeconomic and educational level. Available data in Sri Lanka, though lacking, suggests the annual incidence of sexual abuse to be more than 6000 and that $40.7 \%$ of parents have physically abused their children in the previous month (3).

Significant media attention to matters relating to child abuse and neglect over the last few months have given the impression that child abuse and neglect have recently increased in Sri Lanka. While true increase in the incidence cannot be ascertained, potential causative factors such as mothers leaving for overseas employment, internal migration of parents due to economic reasons and lack of support from extended family have become common in the country. Sri Lankans' increasing preoccupation of success and achievement in both adults and children have reduced the quality of child-parent relationship, making children more vulnerable. Poor parenting skills, serious marital issues, domestic violence, paternal alcohol misuse, poverty, lack of education, social isolation, untreated mental illness in parents and acceptance of the use of violence and force in disciplining children have been identified as causes for child maltreatment which are not uncommon in Sri Lanka (4).

The effects of child maltreatment are profound and varied, with significant social and economic costs (5). Deleterious consequences can be both short-term and long-term. These include academic problems, interpersonal problems, cognitive impairment, obesity, chronic pain, aggression, suicidal behaviour and psychiatric disorders such as depression, anxiety, posttraumatic stress disorder, conduct disorder, eating disorders, personality disorders and substance misuse $(5,6)$. It has been reported that the life time prevalence of psychiatric diagnoses is significantly higher in those who have experienced child abuse when compared to those have not (7). A history of abuse during childhood increases risk of re-victimisation later in life by almost four folds and can negatively affect their ability to be successful parents $(6,8)$. Further, experience of abuse as a child increases the risk of becoming a perpetrator in child abuse, though there is no inevitability about this transition (9). Not all children who are abused develop harmful effects. Protective or resilience factors include the degree of support available factors related to the abuse itself, and peer relationships $(8,9)$.

Preventive efforts, timely intervention and fostering resilience in children may lessen incidence of child abuse and its harmful effects (10). Considering the impact and long standing nature of deleterious effects of child maltreatment and difficulty in treating those effects in some patients, importance of prevention before occurrence cannot be overemphasised. Prevention involves both the use of universal interventions aimed at ensuring protection of children in general and use of targeted interventions in at-risk children $(10,11)$. Latter include alertness by psychiatrists to all the risk factors such as domestic violence, alcohol misuse and mental illness in parents $(10,11)$. The majority of parents with such problems do not abuse their children. However, unless this possibility of such harm is borne in mind, it is unlikely to be recognised or prevented. Universal preventive interventions include mass media and school based programmes to increase awareness about child abuse and teach children about body ownership, the distinction between good and bad touch, recognition of high risk or abusive situations, say 'no,' and disclose abuse to a trusted adult $(10,11)$. Psychiatrists are expected to take leadership in such population preventive strategies which have shown to be generally effective in the prevention of child maltreatment (10).

Early identification is the essential step in breaking the cycle of abuse (10). Psychiatrists need to be vigilant to the possibility of child abuse in any assessment of psychological and behavioural conditions in childhood and in the presence of risk factors. Child protection and intervention for child abuse essentially requires a coordinated multidisciplinary and multi-sectorial approach. Therefore, psychiatrists should collaborate with paediatricians, forensic medicine specialists, gynaecologists, venereologists, police and probation officers in managing children presenting with abuse. Psychiatrists should be aware of the local arrangements of such multi-sectoral management approaches. National guidelines for the management of child abuse and neglect formulated by Colleges of Paediatricians, Psychiatrists and Forensic Pathologists detail inter-disciplinary and 
multi-sectorial approaches such as 'clinical case conference' among clinical specialties within 24 hours of the child presenting to the hospital and 'institutional case conference' with all the relevant agencies including police, probation officers, public health nurse and school when appropriate (12). Psychiatrists or paediatricians are expected to chair the latter. Psychiatrists take the clinical leadership in psychosocial rehabilitation and follow-up of these children. Eclectic approaches informed by cognitive behavioural therapy for children and parents have been found to be effective in mitigating short term and long term harmful effects of child abuse (13). Psychiatrists are uniquely positioned to educate policy makers and those working in child welfare, child health care, and the judicial system about the complex needs of children exposed to child abuse.

The significant involvement of psychiatrists in prevention and management of child abuse underscore the necessity for all psychiatrists to receive specific training in child protection. Psychiatrists could and should play a central role in making Sri Lanka a better place for children of our nation.

A Rodrigo, Department of Psychiatry, University of Kelaniya, Ragama

N Liyanage, University Psychiatry Unit, Colombo North Teaching Hospital, Ragama

Corresponding author: Asiri Rodrigo

E-mail: asirir2000@yahoo.com

Dol http://dx.doi.org/10.4038/sljpsyc.v6i2.8072

\section{References}

1. Butchart A, Harvey AP. Preventing child maltreatment: a guide to taking action and generating evidence. Geneva: World Press; 2006. Available from: http:/whqlibdoc. who.int/publications/2006/9241594365_eng.pdf.

2. Moeller TP, Bachmann GA, Moeller JR. The combined effects of physical, sexual, and emotional abuse during childhood: long-term health consequences for women. Child Abuse Negl 1993; 17(5): 623-40.
3. United Nations Children's Fund (UNICEF). Violence against children. Colombo: UNICEF. 2014 Available from: http://www.unicef.org/srilanka/VAC(3).pdf

4. Department of human services. Causes of child abuse. Melbourne: State government of Victoria; 2011. Available from: http://www.dhs.vic.gov.au/for-individuals/children,families-and-young-people/child-protection/Reporting Child Abuse /what-is-child-abuse/what-are

5. Currie J, Widom CS. Long-term consequences of child abuse and neglect on adult economic well-being. Child Maltreat 2010; 15(2): 111-20.

6. Norman RE, Byambaa M, De R, Butchart A, Scott J, Vos $\mathrm{T}$. The long-term health consequences of child physical abuse, emotional abuse, and neglect: a systematic review and meta-analysis. PLoS Med 2012; 9(11): e1001349.

7. Martin G, Bergen HA, Richardson AS. Sexual abuse and suicidality: Gender differences in a large community sample of adolescents. Child Abuse Negl 2004; 28(5): 491-503.

8. Fillipas HH, Ullman SE. Child sexual abuse, coping responses, self-blame, post-traumatic stress disorder and adult sexual revictimization. J Interpers Violence 2006; 21 (5): $652-72$

9. Sapp MV, Vandeven AM (2005). Update on childhood sexual abuse. Current Opinion in Pediatrics 2005, 17, 25864.

10. Mikton C, Butchart A. Child maltreatment prevention: a systematic review of reviews. Bull World Health Organ. 2009; 87(5): 353-61.

11. Prinz RJ, Sanders MR, Shapiro CJ, Whitaker DJ, Lutzker JR. Population-based prevention of child maltreatment: the U.S. Triple P system population trial. Prev Sci 2009; 10(1): $1-12$

12. Sri Lanka College of Paediatricians (SLCP). National guidelines for the management of child abuse and neglect. Colombo: SLCP; 2014.

13. King NJ, Tonge BJ, Mullen $\mathrm{P}$, et al. Treating sexually abused children with posttraumatic stress symptoms: A randomized clinical trial. J Am Acad Child Adolesc Psychiatry 2000; 39(11): 1347-55. 